\title{
Path integration in 3D from visual motion cues: A human fMRI study
}

\author{
Iole Indovina $^{\mathrm{a}, \mathrm{b}, *}$, Vincenzo Maffei ${ }^{\mathrm{b}}$, Elisabetta Mazzarella ${ }^{\mathrm{b}}$, Valentina Sulpizio ${ }^{\mathrm{c}, \mathrm{d}}$, \\ Gaspare Galati ${ }^{\text {c,d }}$, Francesco Lacquaniti ${ }^{\text {a,b,e }}$ \\ a Centre of Space BioMedicine, University of Rome Tor Vergata, Rome, Italy \\ b Laboratory of Neuromotor Physiology, IRCCS Santa Lucia Foundation, Rome, Italy \\ ${ }^{\mathrm{c}}$ Laboratory of Cognitive and Motor Neurorehabilitation, IRCCS Santa Lucia Foundation, Rome, Italy \\ d Department of Psychology, University of Rome La Sapienza, Rome, Italy \\ e Department of Systems Medicine, University of Rome Tor Vergata, Rome, Italy
}

\section{A R T I C L E I N F O}

\section{Article history:}

Received 29 April 2016

Accepted 4 July 2016

Available online 6 July 2016

\begin{abstract}
A B S T R A C T
While neural correlates of path integration on a yaw plane have been studied extensively, much less is known about path integration in three-dimensions (3D). Here we used fMRI during virtual navigation within tunnels in pseudo-3D. We found that the same visual motion stimuli are encoded differently in the brain depending on whether they represent displacements within the yaw plane or within the pitch plane. The yaw plane is more represented in the hippocampus while the pitch plane is more represented in the angular gyrus (AG) and in the posterior inferior temporal gyrus (pITG), known to be involved in 3D space encoding. In addition, a region in pITG, located just above the previous one, showed two different patterns with multi-voxel analysis, separately coding for the pitch and yaw planes. These results suggest that information encoded within pITG about the yaw plane may be exchanged with the hippocampus, while information about the pitch plane may be exchanged with the AG.
\end{abstract}

(c) 2016 Elsevier Inc. All rights reserved.

\section{Introduction}

In humans and other mammals, navigation relies heavily on the analysis of spatial cues in the plane of locomotion, which is typically taken as the horizontal plane (Ekstrom et al., 2003; Hartley et al., 2014; Iglói et al., 2010; Wolbers et al., 2007). However, movements generally unfold in 3D, and all three axes of movement are important when animals try to orient themselves in 3D while foraging up and down a hill, or when humans explore a cave or tunnel. In these and other cases, neglecting the vertical component of motion would result in a misestimate of the motion path (Jeffery et al., 2013). As navigation problems become much more complex in 3D than 2D (Stella and Treves, 2015), it was suggested that 3D space is represented in a quasi-planar fashion, with space in the horizontal plane being computed separately and represented differently from that along the orthogonal axis (Jeffery et al., 2013). Indeed, a previous study on rats found that, during $3 \mathrm{D}$ navigation, encoding of the vertical direction in the place and grid cells of the hippocampal formation was less accurate than that of the horizontal direction (Hayman et al., 2011), and a computer simulation study showed that the regular tessellation shown by grid cells in $2 \mathrm{D}$

\footnotetext{
* Corresponding author at: Laboratory of Neuromotor Physiology, IRCCS Santa Lucia Foundation, via Ardeatina 306, 00179 Rome, Italy.

E-mail address: i.indovina@hsantalucia.it (I. Indovina).
}

might not be routinely established in 3D (Stella and Treves, 2015). However, other studies in rats (Taube and Shinder, 2013) and monkeys (Orban, 2013) questioned the hypothesis that navigation skills are less reliable in the vertical direction.

One possibility is that the third dimension may be processed in regions other than the hippocampal formation in the vertebrate brain. The posterior parietal cortex (PPC) and inferior temporal gyrus (ITG) appear to be ideal candidates for such encoding, given their role in 3D shape and space processing (Andersen and Buneo, 2002; Galati et al., 2010; Georgieva et al., 2009, 2008: Janssen et al., 2003, 2001, 2000a, 2000b, 1999; Liu et al., 2004; Orban, 2013; Peuskens et al., 2004; Vanduffel et al., 2002). The PPC is an important region for encoding heading direction and navigation in rats (Calton and Taube, 2009; Kesner, 2009; Nitz, 2009; Save and Poucet, 2009; Whitlock et al., 2012), monkeys (Crowe et al., 2005, 2004; Orban, 2013; Phinney and Siegel, 2000; Sakata and Kusunoki, 1992), and in humans particularly in the angular gyrus (AG) (Chrastil et al., 2015; Gourtzelidis et al., 2005; Hummel and Flanagin, 2015; Kravitz et al., 2011; Maguire et al., 1998; Peuskens et al., 2001). In monkeys, neurons for estimating 3D slopes have been found in PPC (Taira et al., 2000; Tsutsui et al., 2002), and TE (Liu et al., 2004) whose putative human homologue for 3D structure extraction appears to be the posterior inferior temporal gyrus (pITG) (Georgieva et al., 2009, 2008; Peuskens et al., 2004). TE was also associated with encoding of 3D environmental shapes aligned with gravity (Vaziri et al., 2014; Vaziri and Connor, 2016). 
Some previous fMRI findings in humans have suggested a selectivity for the visual vertical direction within PPC and PITG, and for the horizontal direction in the hippocampus (Boccia et al., 2015; Indovina et al., 2013b; Shelton and Gabrieli, 2002). In particular, the posterior division of IT and the AG were found to be activated more during encoding and recall of paths from a survey perspective than from a route perspective (Boccia et al., 2015; Shelton and Gabrieli, 2002). During simulated passive transport on a rollercoaster along virtual directions defined by the visual scene, the PPC was more activated during vertical than horizontal motion, and vice versa the hippocampus was more activated during horizontal than vertical motion (Indovina et al., 2013b). However, this study involved high speed accelerated and decelerated motions, unusual in navigation, and did not include a typical navigation task, as path integration, requiring the establishment of a metric map of space (Hartley et al., 2014; Wolbers et al., 2007).

The aim of the current study is to address the issue of 3D space encoding during virtual navigation using univariate and multivariate pattern analyses on fMRI signal. To this end, we used a path integration protocol reminiscent of that of Wolbers et al. (2007), but extended to 3D space. Path integration refers to the ability of humans and other animals of integrating motion cues over time, so as to gather an estimate of the traversed path and displacement in the absence of external landmarks (Etienne and Jeffery, 2004). Several motion cues from the visual, vestibular and somatosensory systems play a role in path integration (Knight et al., 2014). Here, we simulated self-motion by means of purely visual stimuli, i.e. optic flow. The path to be traveled was predetermined in order to ensure identical travel durations across participants and conditions, and to avoid confounding motor activation related to the use of a manipulandum for active drive (Wolbers et al., 2007).

While lying in the fMRI scanner, participants viewed a virtual environment in a first-person perspective, which simulated a travel on a train car moving within tunnels of variable length and orientation. Each trial included the start at a station, where oriented elements in the visual scene (e.g. the floor and ceiling) defined a virtual reference frame so that the visual downward direction was toward the subject's feet, aligned with the body axis. Subsequently, the path pitched upward or downward, or yawed leftward or rightward, and then it continued as a rectilinear segment. Visual stimulation during the rectilinear displacement was the same independently of the preceding curve. At the end of each trial, participants were requested to align with a joystick a 3D arrow (a cone) to the straight line connecting the point of arrival to the origin of the path, thus corresponding to the shortest Euclidean distance between these two points.

Through the univariate analysis, we tested the specific hypotheses that the hippocampus was more activated during virtual displacements in the yaw (axial) plane, while the AG and pITG were more activated during displacements in the pitch (sagittal) plane. Through the multivariate analysis, we further tested whether different patterns of activity encoded these displacements. We also reported data from optic flow stimulation compatible with self-motion that does not imply navigation nor require path integration (Cardin and Smith, 2010; Pitzalis et al., 2010; Wall and Smith, 2008). The aim is to show that together with classically reported areas, also pITG shows a role in self-motion encoding, additional to the static encoding of 3D space.

\section{Methods}

\section{Participants}

20 right-handed participants ( 10 females, 10 males; $22 \pm 3$ years old, mean $\pm \mathrm{sd}$ ) gave written informed consent to participate in this study, which was approved by the Research Ethics Committee of the Santa Lucia Foundation in Rome, according to the Helsinki declaration (http://www.wma.net/en/30publications/10policies/b3/). All participants were right-handed, as assessed via the Edinburgh Handedness Inventory (Oldfield, 1971) and participated in a training and a scanning session. During the training session they learned to perform the task in front of a computer ( 27 trials). They received verbal feedback about whether the response was along the correct Cartesian semi-axis, but not about accuracy in estimation of turning degrees.

We asked participants about sensations they experienced during the simulation both after the training and the scanning session and none of them reported vertigo.

\section{Visual stimuli}

Displacements on a train car were simulated by displaying firstperson perspective views of animated visual scenes compatible with forward self-motion (see Fig. 1 and online material for examples of the stimuli) constructed using commercial software (www. nolimitscoaster.com, Mad Data, Joerg Henseler, Erkrath, Germany). AVI videos were displayed through a MR compatible monitor (BOLDscreen 32 in. LCD, Cambridge Research Systems, http://www. crsltd.com) positioned at the back of the scanner bore by means of Presentation 14.1 (Neurobehavioral Systems Inc., Albany, Canada) at $1440 \times 900$ pixels, 60 frames per second, and viewed at $27^{\circ} \times 17^{\circ}$ visual angle through a mirror positioned at $45^{\circ}$ on the head coil.

Trials

Each trial started with the car leaving a station and traveling forward for $1 \mathrm{~s}$ along the horizontal direction defined by the visual simulation at a virtual speed of $20 \mathrm{~km} / \mathrm{h}$ and then keeping the Ahead direction (A), or turning Leftward (L curve), Rightward (R curve), Upward (U curve), Downward (D curve) by 45 or $90^{\circ}$. After each curve, a rectilinear segment always followed. Rectilinear segments were identical whether following the curve $(\mathrm{L} / \mathrm{R} / \mathrm{U} / \mathrm{D})$ or not $(\mathrm{A})$. Each trial path was traveled within wooden tunnels whose exit was visually occluded by a black panel (see Fig. 1a and videos in online materials). We had 24 trials for each $\mathrm{L} / \mathrm{R} / \mathrm{U} /$ D condition and 12 trials for the A condition (108 total trials).

To avoid habituation and trial collinearity, we varied traveling speeds (see Table S1). In addition, to avoid predictability and so lapse of attention during the rectilinear segment, in 24 trials the rectilinear segment was followed by another curve, not visible from the preceding segment.

\section{Task}

During visual motion stimulation, participants were instructed to fixate a central circle (about $0.5^{\circ}$ visual angle) superimposed on visual stimuli. Afterward, the task consisted in aligning a 3D arrow to the beeline distance traveled, through a joystick grabbed with the right hand (the joystick was produced by hybridmojo LLC, and was MR compatible, http://www.hybridmojo.com/products/mri/joystick.html). In particular, at the end of each trial participants viewed a black screen displaying the "wait" sign for a variable time ( 2 to $6 \mathrm{~s}$ at $0.5 \mathrm{~s}$ steps, $4 \mathrm{~s}$ average). Then a landscape without railways appeared and a cone at the center of the scene pointed toward the participant. Participants were instructed to point the tip of the cone back to where they remembered it was the origin of each traveled path, in order to align the cone with the beeline, from the point of arrival to the origin of the path, and to push the joystick button to record their choice. In this way, we forced participants to base their judgements on an allocentric perspective, that is, independently of the virtual orientation of the participant body at the end of the travel. In addition, the cone was superimposed on a visual scene defining the virtual 3D external reference frame where the train traveled (landscape), further forcing an allocentric rather than egocentric perspective. Finally, after participants made their choice through the joystick, a black screen appeared until the end of the allowed response time $(5 \mathrm{~s})$. To align it with body axis, the joystick was attached on the vertical surface of a box positioned on the participant belly. Each experiment consisted of 4 sessions, each including 27 trials (108 total trials). Each session lasted about 8:20 min (260 scans). 

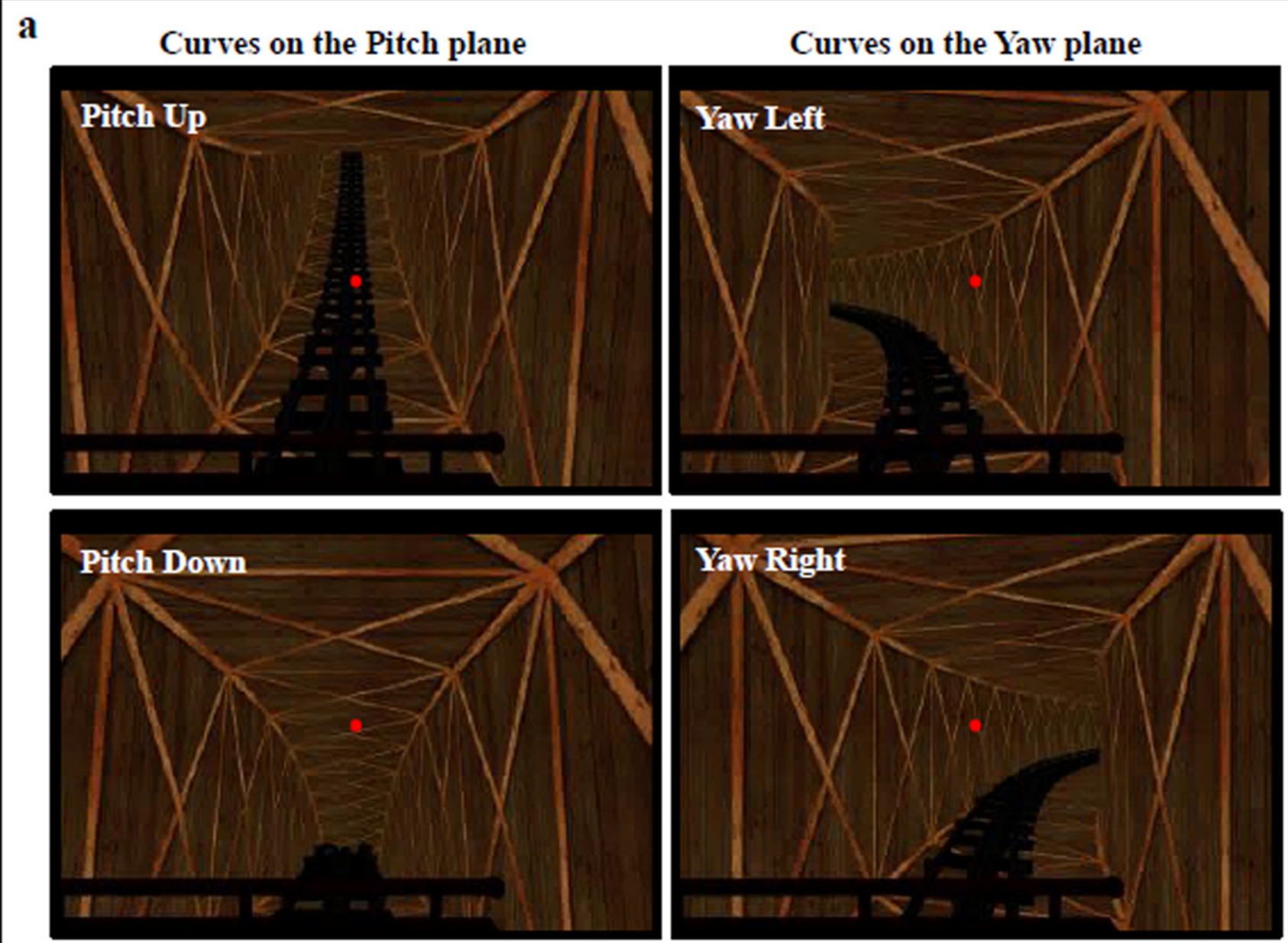

b

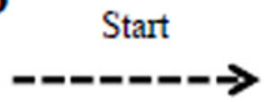

$1 \mathrm{~s}$

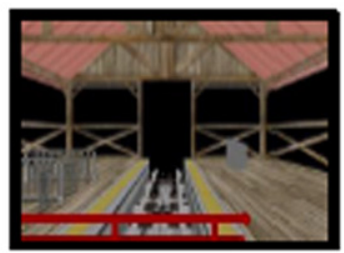

Rectilinear displacement on pitch or yaw plane

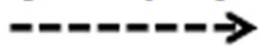

2 to $7.2 \mathrm{~s}$

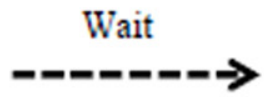

2 to $6 \mathrm{~s}$
Align the cone

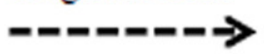

$5 \mathrm{~s}$
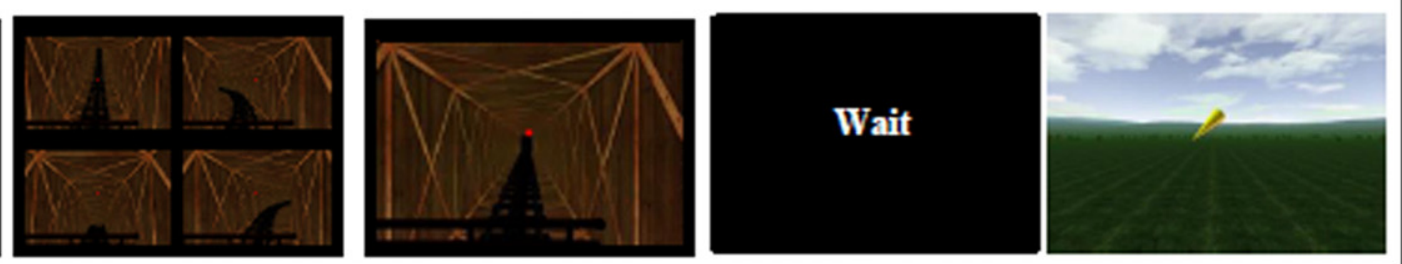

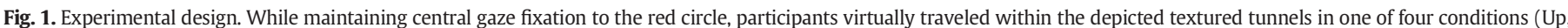

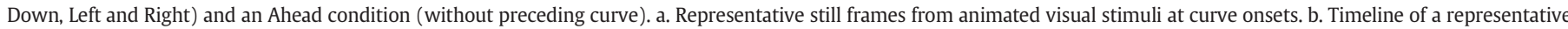

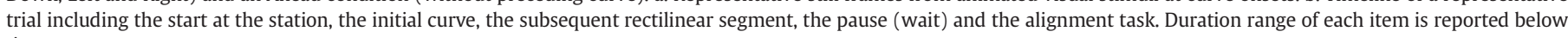
time arrows.

The choice of an allocentric perspective was made in order to overcome difficulties related to spatial updating of self-orientation in the virtual environment (Riecke et al., 2012), particularly in the scanner where the subject lay supine, an unusual position for navigation. Also previous studies on 3D textured tunnel navigation used this shift from an egocentric perspective during visual stimulation to an allocentric perspective during response (Vidal et al., 2006, 2004, 2003; Wolbers and Büchel, 2005). In general, it is thought that the study of navigation through self-motion cues (idiothetic navigation) in the fMRI scanner has inherent drawbacks due to the lack of dynamic vestibular, proprioceptive and tactile cues in conflict with dynamic visual motion cues, in contrast with real navigation where such cues are generally congruent (Riecke et al., 2012; Taube et al., 2013). However, in the present study, we were interested in the aspects of 3D path integration related to visual cue encoding, independently of other sensory modalities, and these aspects were extensively investigated in path integration studies on 2D paths (Chrastil et al., 2015; Sherrill et al., 2013; Wolbers et al., 2007; Wolbers and Büchel, 2005).

\section{Self-motion compatible optic flow}

In order to localize regions related to simulated self-motion, we used optic flow self-motion compatible stimulation (Cardin and Smith, 2010; Pitzalis et al., 2010; Wall and Smith, 2008). This consisted of 2 time- 
varying optic flows (light dots on a dark background) representing self and object random motion to be compared, generated by X11/OpenGL software (Dale and Sereno, 1993). The first self-motion compatible optic flow (self-motion condition) consisted of 16-s blocks of coherent dot field motion that simulated motion of the observer. A new field of white dots was generated every $500 \mathrm{~ms}$ (dot size $0.4^{\circ} \times 0.4^{\circ}$ ). Dots immediately began to move along a trajectory so as to generate a coherent movement on a plane. The motion pattern was chosen randomly for that 500 -ms period from a continuum ranging from dilation to outward spiral, to rotation, to inward spiral, to contraction. The center of the movement was jittered from flow to flow, and the speed varied within a small range. During the scrambled period (random condition), dots and their movement vectors were generated as during the self-motion condition except that each dot trajectory was rotated by a random angle around the pattern center before execution. This procedure scrambled the coherency of movement (at a given point, dots moved in different directions) but preserved the speed gradient (central dots still moved slower than peripheral dots). Eight blocks of $16 \mathrm{~s}$ were presented for the self and random conditions and were repeated in two sessions of $4: 16$ min each.

\section{fMRI}

We acquired GE-EPI images on 3T Philips Achieva scanner (8 channels SENSE head coil, TR $=2 \mathrm{~s}$, TE $=25 \mathrm{~ms}$, flip angle $=80^{\circ}$, 39 slices, $2.5 \mathrm{~mm}$ slice thickness, $1.25 \mathrm{~mm}$ slice gap, $3.59 \times 3.59 \times 3.75$ voxel size, ascending acquisition, $230 \times 230 \times 145 \mathrm{~mm}$ FOV). We acquired 4 EPI sessions of 260 scans each for the navigation experiment and 2 EPI sessions of 138 scans each for the self-motion compatible optic flow.

\section{Analysis}

\section{Behavioral data analysis}

The ability to discriminate direction was assessed by computing the percentage of correct button-press responses in each condition (U, D, L, $\mathrm{R}, \mathrm{A}$ ), that is, responses that occurred on the expected Cartesian semiaxis, arcsine transformed to make their distribution normal prior to statistical tests. Those trials presenting additional curves to avoid predictability (see Trials) were excluded from the analysis.

Turning degrees were also analyzed. The direction of the arrow during correct trials was converted to radians for repeated measures analysis of variance (RM-ANOVA). RM-ANOVA was performed on (Greenhouse-Geisser corrected) average direction of the arrow in correct trials, with direction (pitch/yaw), turning degrees (45/90) and speed on curves $(20 / 50 / 80 \mathrm{~km} / \mathrm{h})$ as within-subject factors.

\section{fMRI Analysis}

\section{Univariate analysis}

FMRI data preprocessing was carried out using FEAT (FMRI Expert Analysis Tool) Version 5.98, part of FSL (FMRIB's Software Library, www.fmrib.ox.ac.uk/fsl). Three volumes were discarded at the beginning of each session to allow T1 decay. The following pre-statistics processing was applied: motion correction using MCFLIRT (Jenkinson et al., 2002); spatial smoothing using a Gaussian kernel of FWHM 8 mm; grand-mean intensity normalisation of the entire 4D dataset by a single multiplicative factor; high-pass temporal filtering (Gaussian-weighted least-squares straight line fitting, with high pass filter at $60 \mathrm{~s}$ for event related design during the path integration protocol and $120 \mathrm{~s}$ for block design during the self-motion optic flow protocol). Time-series statistical analysis was carried out using FILM with local autocorrelation correction (Woolrich et al., 2001). Motion parameters were entered in the model as covariates of no interest. The group analysis was carried out using FLAME (FMRIB's Local Analysis of Mixed Effects) (Beckmann et al., 2003; Woolrich, 2008; Woolrich et al., 2004).

For the self-motion optic flow protocol, self and random conditions were modeled as blocks of $16 \mathrm{~s}$ and the self-motion versus randommotion condition contrast was analyzed.

For the path integration task, 16 conditions were modeled: $U / D / L / R$ curves, $\mathrm{A} / \mathrm{U} / \mathrm{D} / \mathrm{L} / \mathrm{R}$ rectilinear segments and waiting periods separately, joystick movement and last additional curves (see "Trials" paragraph). The main contrasts of interest included rectilinear segment comparison during yaw plane $(L, R)$ versus pitch plane $(U, D)$ visual motion and pitch versus yaw plane visual motion. These comparisons did not include the A trials.

\section{Multivariate analysis}

A complementary analysis to study the segregation of the neural representation for pitch and yaw plane displacement was based on a multivariate classification analysis, where a classifier was trained to discriminate multi-voxel patterns of estimated BOLD responses to pairs of items (i.e., pairing of left, right, up, down directions) (Norman et al., 2006). A searchlight analysis (Kriegeskorte et al., 2006) was implemented using custom MATLAB code (The MathWorks Inc., Natick, MA, USA) to look for areas with high classification accuracy within the entire brain volume. A small spherical region of interest (ROI) (diameter $9 \mathrm{~mm}$ ) was created and centered on each voxel of the grey matter in turn, classifying the patterns extracted from that ROI, and assigning the classification outcome score to the ROI central voxel, thus creating a whole-brain image of classification success.

We first ran a General Linear Model (GLM) on unsmoothed preprocessed images of each fMRI run where each motion orientation (i.e., left, right, up, down) was modeled as a different experimental condition. This yielded a voxel-by-voxel estimate (i.e., an image) of the amplitude of the BOLD response evoked by each of the 4 trial types within each run. Classification was then performed on multi-voxel patterns extracted from these images. We assigned each image to either of two categories, representing the pitch and yaw planes, respectively. Then, we trained a linear discriminant analysis (LDA) classifier to learn the association between the category and the corresponding images. This was achieved by splitting the data set into a training set and a test set. We used a leave-one-out cross-validation procedure to test classification outcomes on a data set independent from that used for training the classifier: images from all runs except one were used in turn to train the classifier and the remaining data were used to evaluate prediction accuracy. The resulting classification outcomes were averaged across crossvalidation folds. This procedure was repeated for each searchlight sphere, thus generating an accuracy value (D-prime score) for every single voxel within the search space. These values were then used to create subject-specific accuracy maps which were entered into a second-level random effects analysis.

\section{Statistical significance}

To assess the statistical significance at group level of the univariate and multivariate analyses both a priori ROI and whole-brain analyses were performed.

For the region of interest ROI approach we controlled for Family Wise Error (FWE) within the union of the ROIs (small volume correction, svc) (Friston, 1997; Worsley et al., 1996). Z-statistic images were thresholded using Gaussian Random Field -theory-based maximum height thresholding with a svc corrected significance threshold of $p<0.05$ (Worsley, 2001).

For the whole-brain analysis, we controlled for FWE at cluster level using the whole brain as volume of interest. $\mathrm{Z}$ statistic images were thresholded using default values set in FSL, namely cluster minimum size determined by $p<0.01$ and a whole brain corrected cluster significance threshold of $p<0.05$ (Worsley, 2001). However, in light of recent 
suggestions that a more conservative threshold should be used for the whole-brain fMRI analyses (Woo et al., 2014), we also applied a more conservative voxelwise threshold of $p<0.001$ to determine minimum cluster size.

\section{Regions of interests (ROIs)}

Based on human and animal literature, we had strong a priori hypotheses that the hippocampus contributes to coding of yaw more than pitch plane displacements and pITG and AG of pitch more than yaw plane displacements (Hayman et al., 2011; Indovina et al., 2013b; Orban, 2013; Vaziri and Connor, 2016).

Then, regions of interest included the bilateral hippocampus for the contrast yaw > pitch plane displacement; bilateral AG and bilateral pITG for the contrast pitch $>$ yaw plane displacement. All ROIs were defined anatomically according to the automated anatomical labeling atlas (aal) (Tzourio-Mazoyer et al., 2002). pITG was derived by restricting the aal inferior temporal gyrus ROI to the grey matter adjacent to the posterior inferior temporal sulcus in caret (Van Essen, 2005).

\section{Results}

\section{Behavioral results}

Participants responded correctly on average in $94 \%$ of trials, ranging from $76 \%$ correct answers for the worst participant to $100 \%$ for the best participant. Number of correct answers did not differ across genders (two-sample $t$-test, $t(18)=0.4, p=0.7$ ), pitch and yaw planes (paired $t$-tests, $t(19)=1.3, p=0.2)$, left and right orientations $(t(19)=0.2$, $p=0.8$ ), up and down orientations ( $t(19)=0.5, p=0.6)$, nor between all these conditions and the ahead condition $(t(19)=1.7, p=0.1)$.

RM-ANOVA on direction of the arrow did not show an effect of plane (pitch/yaw) $(\mathrm{F}(1,19)=1.8, p=0.2)$, nor of speed on curves $(\mathrm{F}(2,38)=$ $2.4, p=0.1)$. It showed a significant, though small, effect of turning degrees $(\mathrm{F}(1,19)=5.0, p=0.04)\left(\right.$ curve $45^{\circ}=29^{\circ} \pm 5^{\circ}$, curve $90^{\circ}=$ $31^{\circ} \pm 5^{\circ}$; mean $\left.\pm \mathrm{sem}\right)$. These results indicate that participants were unable to finely estimate degrees of turning, but they clearly distinguished between orientations.

\section{fMRI results}

\section{Self-motion compatible optic flow}

Several regions encompassing the superior and middle occipital gyrus, fusiform and lingual gyrus, inferior temporal gyrus, superior parietal lobe, cuneus, vermis and cerebellum were activated ( $p$-FWE whole brain cluster level corrected $<0.05$, at both $p<0.01$ and $p<0.001$ for minimum cluster size, Table S2, Fig. S1). These areas comprised those reported in the literature as putative V6 (pV6) and putative ventral intraparietal area (pVIP) (Cardin and Smith, 2010; Pitzalis et al., 2010), and additionally pITG.

\section{Path integration}

\section{Univariate analysis}

\section{All rectilinear motions}

All rectilinear motions (Pitch + Yaw) were associated with widespread activations (Fig. S2, Table S3, p-FWE whole brain cluster level corrected $<0.05$, at both $p<0.01$ and $p<0.001$ for minimum cluster size) in brain regions processing visual motion (lateral occipitotemporal cortex including putative human visual motion complex hMT/V5 +, parietal cortex including putative VIP, parieto-occipital sulcus including putative V6, cingulate sulcus regions), sensorimotor activity (pre-central gyrus and sulcus, SMA, cerebellum), or arousal (anterior insula) (Eckert et al., 2009). These sites of activation correspond to those previously reported with other types of optic flow stimuli (Indovina et al., 2013b).

Yaw plane $>$ pitch plane displacement

At $p<0.05$ svc (ROI analysis) this comparison activated bilaterally the hippocampus (left hippocampus xyz $=-28-4-22$, z-score $=3.2$, $\mathrm{p}$-corr $=0.02$; right hippocampus xyz $=20-6-16$, z-score $=3.0$; $\mathrm{p}$ corr $=0.04$ ) (Fig. 2).

At whole brain level ( $\mathrm{p}-\mathrm{FWE}$ whole brain cluster level corrected $<0.05, p<0.01$ for minimum cluster size), the superior frontal gyrus, precentral gyrus regions and supplementary motor area (SMA) were activated (table S4). As this last result did not survive the whole-brain more conservative threshold ( $p<0.001$ for minimum cluster size), we did not further discuss it.

\section{Pitch plane $>$ yaw plane displacement}

At $p<0.05 \mathrm{svc}$ (ROI analysis) this comparison activated the posterior portion of right ITG and a region in the AG (MNI coordinates: right pITG $\mathrm{xyz}=56-54-16, \mathrm{z}$-score $=3.7, \mathrm{p}$-corr $=0.01$; right $\mathrm{AG} \mathrm{xyz}=36-72$ 50 , z-score $=3.3$, p-corr $=0.04$ ) (Fig. 3 ).

At whole brain level ( $\mathrm{p}$-FWE whole brain cluster level corrected $<0.05, p<0.01$ for minimum cluster size), a right parietal/occipital region was activated, with the maximum activity on the AG reported above from the ROI analysis, and encompassing posteriorly the superior occipital gyrus and anteriorly the intraparietal sulcus and supramarginal gyrus. This last result did not survive the whole-brain more conservative threshold ( $p<0.001$ for minimum cluster size).

\section{Effect of orientation}

Up vs Down and Right vs Left orientation comparisons did not elicit any statistically significant activation.

\section{Multivariate analysis}

Voxel pattern activity conveyed distinct representations of pitch and yaw planes in the right pITG ( $\mathrm{p}$-FWE whole brain cluster level corrected $<0.05$, at both $p<0.01$ and $p<0.001$ for minimum cluster size; MNI coordinates: $x y z=50-54-4$, z-score $=4.7$ ). This pattern of activity was found in a cluster superiorly adjacent to the pITG cluster showing preference for the pitch plane in the univariate analysis (Fig. 4). This region was within the PITG ROI. No further regions survived at svc significance level.

\section{Discussion}

The current results show that during path integration, identical visual stimuli representing self-motion within the pitch plane are differentially encoded with respect to those within the yaw plane in PITG, the AG and the hippocampus. In particular, one region within PITG and another one within AG preferentially encode virtual displacements in the pitch plane. In addition, a second region within PITG, adjacent but distinct relative to the previous one, shows two populations of voxels that encode for the pitch and yaw plane, respectively. Conversely, the hippocampus preferentially encodes virtual displacements in the yaw plane. In the hippocampus we failed to find different population of voxels separately encoding for yaw and pitch planes. This last result is in accordance with recent accounts in the rat hippocampus showing encoding of the yaw plane displacement and failure to code for the pitch plane displacement (Hayman et al., 2011).

The hippocampal formation, through specialized cells (place, grid, boundary, head direction cells), is generally associated with the metric computation of a flexible map-like representation (Ekstrom et al., 2003; Hartley et al., 2014; Iglói et al., 2010). We found that planes of displacement (pitch, yaw) but not the orientation of displacement within these planes (left, right, up, down) are differently represented. This is not unexpected as a coherent fMRI signal is derived in the yaw plane 


\section{Yaw plane $>$ Pitch plane rectilinear displacement}
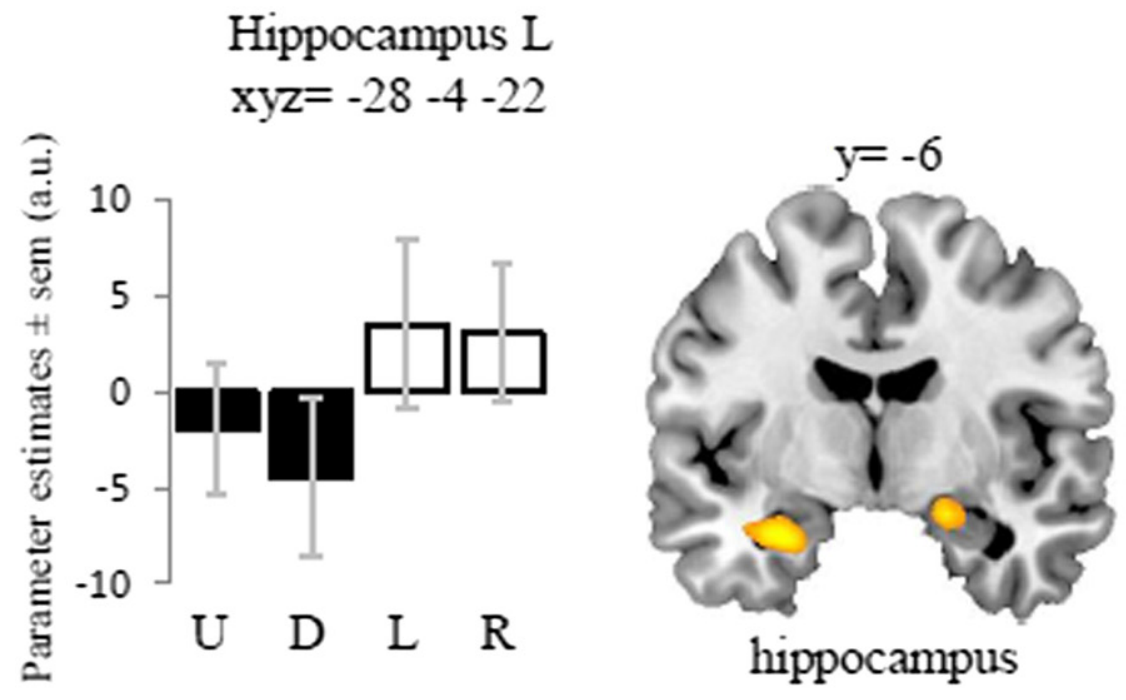

\section{Hippocampus $\mathrm{R}$ $\mathrm{xyz}=20-6-16$}

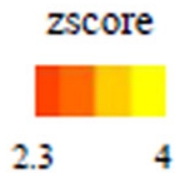

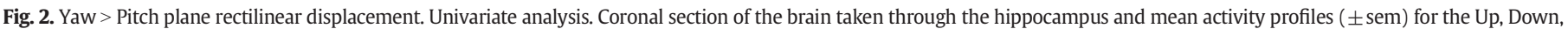

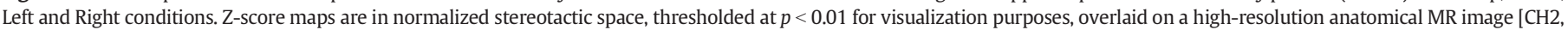
Montreal Neurological Institute (MNI)], with the left side shown on the left.

in response to virtual displacements in the six specific axes that compose the triangular grid characterizing grid cells (Doeller et al., 2010; Horner et al., 2016), rather than in a specific direction. Indeed, with sophisticated analyses, human fMRI studies were able to detect 6-fold rotational symmetry in a network of areas comprising the entorhinal cortex (Doeller et al., 2010; Horner et al., 2016).

The hippocampal formation is generally associated with navigation from both allocentric and egocentric information (Ekstrom et al., 2003; Hartley et al., 2014; Iglói et al., 2010), while the PPC is specifically associated with egocentric navigation (Hartley et al., 2014; Maguire et al., 1998).

The current task required participants to evaluate 3D displacements from optic flow direction during curves, and then to build a 3D map of total displacement in an allocentric reference frame, independently of the relative personal orientation. Though this task presents a mixture of egocentric and allocentric components, and indeed fully egocentric or allocentric navigation is difficult to imagine in the real world, the key point is that the same reference frame is used throughout the task for pitch and yaw plane displacements, thus providing evidence for a differential role of the hippocampus and PPC within the same reference frame.

The novel finding of this study consists in the role of pITG in encoding 3D displacement. One subregion showed distinct voxel populations for either pitch or yaw plane displacements, while another subregion, ventral to the previous one, specifically encoded displacements in the pitch plane. These pITG subregions are just anterior to the human visual motion complex hMT/V5 + and are also activated by the self-motion vs random comparison in the self-motion compatible stimulation (Fig. 4, Fig. S1). It was associated with 3D shape and space processing (Georgieva et al., 2009, 2008; Peuskens et al., 2004; Vanduffel et al., 2002), but to our knowledge it was never before associated with the abstract representation of visual 3D space during simulated displacements on pitch and yaw planes. It has been previously associated with navigation only in a couple of studies, interestingly when a survey rather than a route perspective was assumed (Boccia et al., 2015; Shelton and Gabrieli, 2002). In all these respects it appears to be more at the interface between the dorsal and ventral streams rather than belonging to the ventral stream per se (Goodale and Milner, 1992). It has been hypothesized that pITG is the human homologue of TE (Georgieva et al., 2009). Though TE is located rostrally in the inferior temporal gyrus (Kravitz et al., 2011), this region is strongly associated with 3D shape processing (Janssen et al., 2003, 2001, 2000a, $2000 \mathrm{~b})$. Moreover, though belonging to the ventral stream, TE was found to code for depth from binocular disparity and texture cues (Janssen et al., 2001; Liu et al., 2004; Orban et al., 2006). Most importantly, TE has been associated with encoding of environmental shapes and scene elements that are typically aligned with gravity as ground surfaces and ceilings (Vaziri et al., 2014; Vaziri and Connor, 2016). TE has extensive bidirectional connections, both directly and through TEO, with the posterior parietal cortex and the hippocampal formation, indicating interaction between the ventral and dorsal stream and suggesting a possible role in navigation (Distler et al., 1993; Kravitz et al., 2011; Webster et al., 1994, 1991).

The functional-anatomical gradient in the PITG transitioning from 2D to 3D representation is reminiscent of the path found in the presubiculum of bats (Finkelstein et al., 2015). There, 'classical' head direction cells responding allocentrically to azimuth were found, together with 'pitch' cells tuned to pitch and 'conjunctive' cells tuned to azimuth 


\section{Pitch plane > Yaw plane rectilinear displacement}

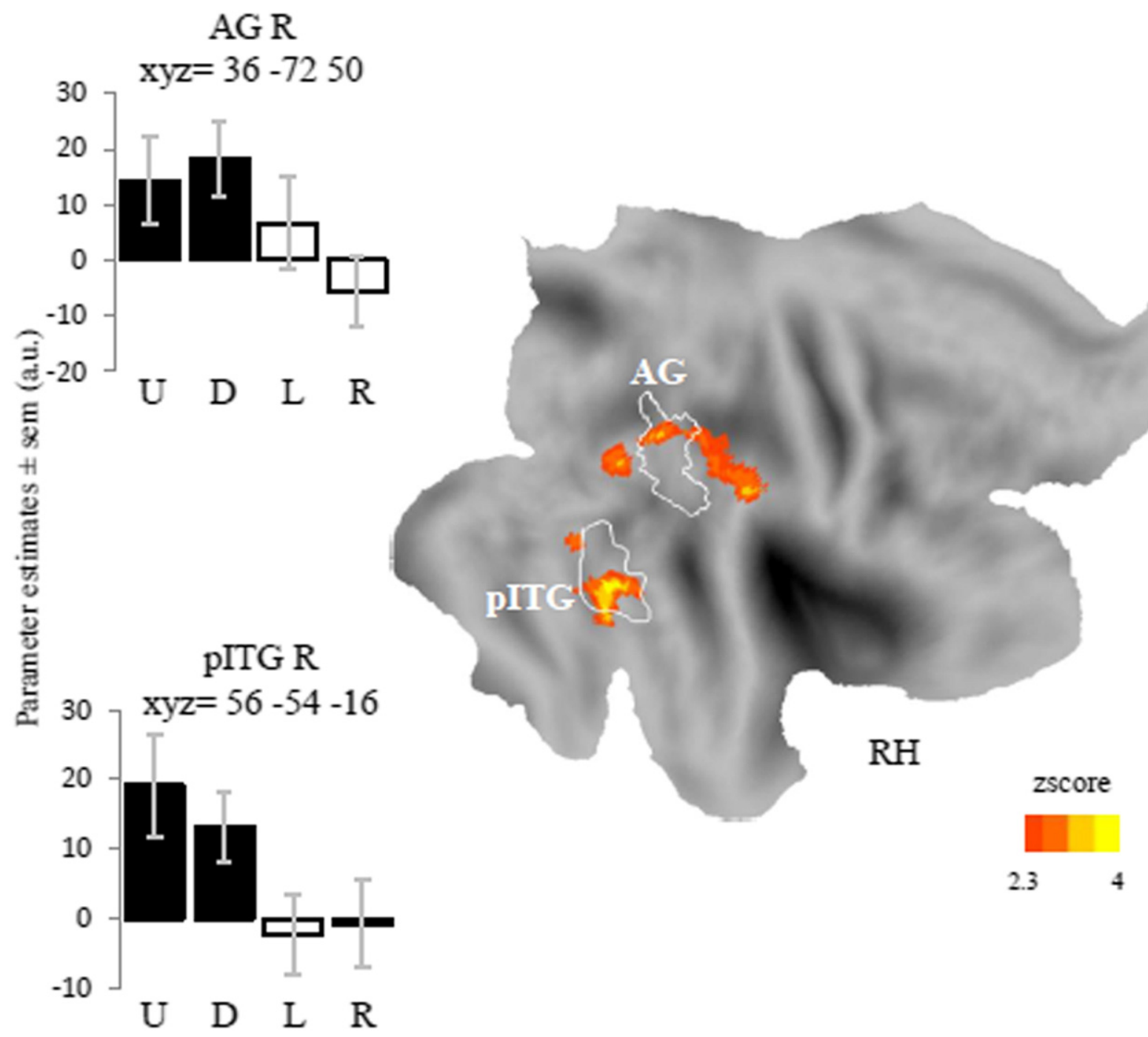

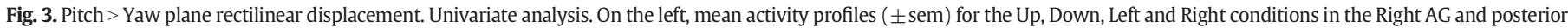

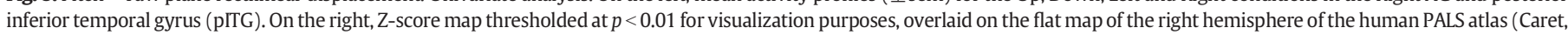
(Van Essen, 2005)). ROIs for the AG and pITG are outlined in white. $\mathrm{RH}=$ right hemisphere.

and pitch (Finkelstein et al., 2015). Pitch cells provided egocentric encoding, while azimuth cell responses were the same whether the bat was upright or upside-down (toroidal coordinate encoding). In bats, this dichotomic signal in the head direction system is transmitted to the hippocampus place cells (Rubin et al., 2014), where volumetric isotropic encoding was found (Yartsev and Ulanovsky, 2013), in contrast with what is found in rats (Hayman et al., 2011).

Differences between bat and rat or human navigation systems are not surprising, as the human and rat systems are mainly adapted to find paths in 2D while bats mainly navigate in 3D open-air environments.

We speculate that top-down signals from the prefrontal cortex, differentiating identical visual motion information based on cognitive cues, reach the pITG, where a 3D representation of space is built and segregated along pitch and yaw planes, and then sent to the AG and the hippocampal formation respectively.

In the hippocampal formation, signals containing information about distance traveled and direction in the yaw plane are used by specialized cells to build a metric map (Hartley et al., 2014). In the posterior parietal cortex and pITG the existence of such kind of 'metric' cells has not been directly reported before. However, the PPC has been shown to encode direction (Fabbri et al., 2010; Gourtzelidis et al., 2005; Peuskens et al., 2001), and speed of optic flow (Phinney and Siegel, 2000), from which traveled distance could be computed. The AG was shown to track homing distance during path integration analogously to the hippocampal formation (Chrastil et al., 2015). Moreover, pITG and the posterior intraparietal sulcus showed a proportional response to the physical distance between consecutive places and views in a repetition suppression protocol (Sulpizio et al., 2014). Finally, grid-cell-like representations in humans were found in posterior and medial parietal areas, lateral temporal and medial prefrontal areas (Doeller et al., 2010).

A role for space anisotropy created by gravity can be hypothesized for the current findings. Indeed, previous studies have outlined the importance of vision to define the perceived direction of gravity even in the face of conflicting vestibular and somatosensory cues (Guerraz et al., 1998; Held et al., 1975; Indovina et al., 2015, 2013a, 2013b; 


\section{Pitch plane > Yaw plane rectilinear displacement}

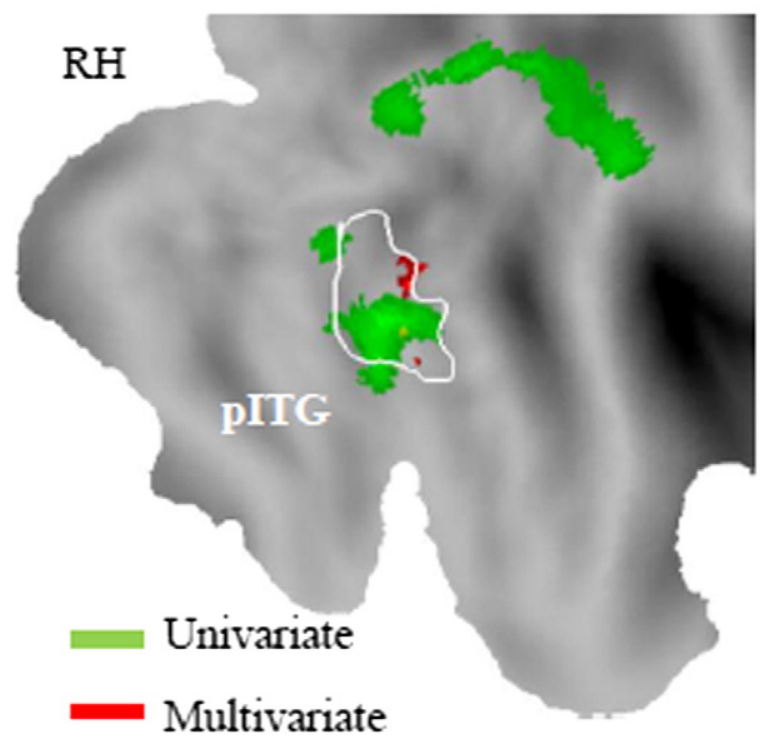

Fig. 4. Pitch vs Yaw plane rectilinear displacement. Voxels decoding pitch and yaw planes significantly better than chance (accuracy map thresholded at $p<0.01$ for visualization purposes) are reported in red (multivariate analysis demonstrating pitch and yaw plane displacement differential encoding), together with the results from the univariate analysis that are reported in green (Pitch > Yaw plane displacement) for comparison. Results are superimposed on the flat map of the right hemisphere of the human PALS atlas (Caret). The ROI for pITG is outlined in white. $\mathrm{RH}=$ right hemisphere.

Lacquaniti et al., 2013; Mast et al., 1999; Vaziri and Connor, 2016; Witkin and Asch, 1948). In the current task, the visual scene cued the direction of a virtual (scene-based) vertical at the beginning (at the train station) and at the end (when an outdoors landscape was shown) of each trial. By means of these reference cues, participants might be led to imagine to move along azimuthal or elevation planes. It is possible that some visual areas in the ventral stream are specialized to recognition of gravitational visual features. Indeed, previous studies showed that the ventral stream recognizes visual gravitational cues representing object weight (Gallivan et al., 2014), and biological motion on Earth rather than Moon (Maffei et al., 2015).

In conclusion, although in the navigation literature the major focus has been on the hippocampal formation, a vast network of additional areas complement its function while also conferring a high degree of redundancy to the navigation system. With different levels of specialization, neurons across various brain regions are tuned by parameters relevant to navigation as self-motion speed and direction. Here we found a specialization of the hippocampus for path integration in the yaw plane, and of PITG and AG in the pitch plane. Partial segregation of information between orthogonal planes may allow parallel processing and simplify spatial navigation processing with respect to volumetric encoding. Whether and how information about displacement in the pitch and yaw planes is integrated to provide a volumetric representation of space remains an open question.

\section{Limitations of the study}

In navigation, both body-based and visual cues contribute to accurate orientation coding. The anterior dorsal nucleus (ADN) of the thalamus is thought to integrate this information (Yoder and Taube, 2014). A recent fMRI study provided body-based cues in a preparatory session outside the scanner, and was thus able to detect signals in the thalamus (Shine et al., 2016). The current study, as most previous human fMRI studies using virtual environments in which only visual cues were provided (Chrastil et al., 2015; Doeller et al., 2010; Iglói et al., 2010; Maguire et al., 1998; Sherrill et al., 2013; Wolbers et al., 2007; Wolbers and Büchel, 2005), limits the investigation to aspects of navigation related to encoding visual cues. Horner and co-workers, however, demonstrated that grid-cell activity may be investigated even through mental imagery (Horner et al., 2016).

\section{Financial disclosures}

None of the authors have conflict of interests.

\section{Acknowledgments}

Our work was supported by the Ministry of Health, Italy (RF-10.057 Grant), Italian Ministry of University and Research (PRIN Grant 2010MEFNF7_002), and Italian Space Agency (COREA Grant 2013084-R.0).

\section{Appendix A. Supplementary data}

Supplementary data to this article can be found online at http://dx. doi.org/10.1016/j.neuroimage.2016.07.008.

\section{References}

Andersen, R.A., Buneo, C.A., 2002. Intentional maps in posterior parietal cortex. Annu. Rev. Neurosci. 25, 189-220. http://dx.doi.org/10.1146/annurev.neuro.25.112701.142922.

Beckmann, C.F., Jenkinson, M., Smith, S.M., 2003. General multilevel linear modeling for group analysis in FMRI. NeuroImage 20, 1052-1063. http://dx.doi.org/10.1016/ S1053-8119(03)00435-X.

Boccia, M., Guariglia, C., Sabatini, U., Nemmi, F., 2015. Navigating toward a novel environment from a route or survey perspective: neural correlates and context-dependent connectivity. Brain Struct. Funct. http://dx.doi.org/10.1007/s00429-015-1021-z.

Calton, J.L., Taube, J.S., 2009. Where am I and how will I get there from here? A role for posterior parietal cortex in the integration of spatial information and route planning. Neurobiol. Learn. Mem. 91, 186-196. http://dx.doi.org/10.1016/j.nlm.2008.09.015.

Cardin, V., Smith, A.T., 2010. Sensitivity of human visual and vestibular cortical regions to egomotion-compatible visual stimulation. Cereb. Cortex N. Y. N 1991 (20), 1964-1973. http://dx.doi.org/10.1093/cercor/bhp268.

Chrastil, E.R., Sherrill, K.R., Hasselmo, M.E., Stern, C.E., 2015. There and back again: hippocampus and retrosplenial cortex track homing distance during human path integration. J. Neurosci. 35, 15442-15452. http://dx.doi.org/10.1523/JNEUROSCI.1209-15. 2015.

Crowe, D.A., Chafee, M.V., Averbeck, B.B., Georgopoulos, A.P., 2004. Neural activity in primate parietal area 7a related to spatial analysis of visual mazes. Cereb. Cortex N. Y. N 1991 (14), 23-34

Crowe, D.A., Averbeck, B.B., Chafee, M.V., Georgopoulos, A.P., 2005. Dynamics of parietal neural activity during spatial cognitive processing. Neuron 47, 885-891. http://dx. doi.org/10.1016/j.neuron.2005.08.005.

Dale, A.M., Sereno, M.I., 1993. Improved localization of cortical activity by combining EEG and MEG with MRI cortical surface reconstruction: a linear approach. J. Cogn. Neurosci. 5, 162-176. http://dx.doi.org/10.1162/jocn.1993.5.2.162.

Distler, C., Boussaoud, D., Desimone, R., Ungerleider, L.G., 1993. Cortical connections of inferior temporal area TEO in macaque monkeys. J. Comp. Neurol. 334, 125-150. http:// dx.doi.org/10.1002/cne.903340111.

Doeller, C.F., Barry, C., Burgess, N., 2010. Evidence for grid cells in a human memory network. Nature 463, 657-661. http://dx.doi.org/10.1038/nature08704.

Eckert, M.A., Menon, V., Walczak, A., Ahlstrom, J., Denslow, S., Horwitz, A., Dubno, J.R., 2009. At the heart of the ventral attention system: the right anterior insula. Hum. Brain Mapp. 30, 2530-2541. http://dx.doi.org/10.1002/hbm.20688.

Ekstrom, A.D., Kahana, M.J., Caplan, J.B., Fields, T.A., Isham, E.A., Newman, E.L., Fried, I., 2003. Cellular networks underlying human spatial navigation. Nature 425, 184-188. http://dx.doi.org/10.1038/nature01964.

Etienne, A.S., Jeffery, K.J., 2004. Path integration in mammals. Hippocampus 14, 180-192. http://dx.doi.org/10.1002/hipo.10173.

Fabbri, S., Caramazza, A., Lingnau, A., 2010. Tuning curves for movement direction in the human visuomotor system. J. Neurosci. 30, 13488-13498. http://dx.doi.org/10.1523/ JNEUROSCI.2571-10.2010.

Finkelstein, A., Derdikman, D., Rubin, A., Foerster, J.N., Las, L., Ulanovsky, N., 2015. Threedimensional head-direction coding in the bat brain. Nature 517, 159-164. http://dx. doi.org/10.1038/nature14031.

Friston, K.J., 1997. Testing for anatomically specified regional effects. Hum. Brain Mapp. 5, 133-136.

Galati, G., Pelle, G., Berthoz, A., Committeri, G., 2010. Multiple reference frames used by the human brain for spatial perception and memory. Exp. Brain Res. Exp. Hirnforsch. Expérimentation Cérébrale 206, 109-120. http://dx.doi.org/10.1007/s00221-0102168-8. 
Gallivan, J.P., Cant, J.S., Goodale, M.A., Flanagan, J.R., 2014. Representation of object weight in human ventral visual cortex. Curr. Biol. CB 24, 1866-1873. http://dx.doi.org/10. 1016/j.cub.2014.06.046.

Georgieva, S.S., Todd, J.T., Peeters, R., Orban, G.A., 2008. The extraction of 3D shape from texture and shading in the human brain. Cereb. Cortex N. Y. N 1991 (18), 2416-2438. http://dx.doi.org/10.1093/cercor/bhn002.

Georgieva, S., Peeters, R., Kolster, H., Todd, J.T., Orban, G.A., 2009. The processing of threedimensional shape from disparity in the human brain. J. Neurosci. 29, 727-742. http://dx.doi.org/10.1523/JNEUROSCI.4753-08.2009.

Goodale, M.A., Milner, A.D., 1992. Separate visual pathways for perception and action. Trends Neurosci. 15, 20-25.

Gourtzelidis, P., Tzagarakis, C., Lewis, S.M., Crowe, D.A., Auerbach, E., Jerde, T.A., Uğurbil, K., Georgopoulos, A.P., 2005. Mental maze solving: directional fMRI tuning and population coding in the superior parietal lobule. Exp. Brain Res. 165, 273-282. http://dx. doi.org/10.1007/s00221-005-2298-6.

Guerraz, M., Poquin, D., Ohlmann, T., 1998. The role of head-centric spatial reference with a static and kinetic visual disturbance. Percept. Psychophys. 60, 287-295.

Hartley, T., Lever, C., Burgess, N., O'Keefe, J., 2014. Space in the brain: how the hippocampal formation supports spatial cognition. Philos. Trans. R. Soc. Lond. Ser. B Biol. Sci. 369, 20120510. http://dx.doi.org/10.1098/rstb.2012.0510.

Hayman, R., Verriotis, M.A., Jovalekic, A., Fenton, A.A., Jeffery, K.J., 2011. Anisotropic encoding of three-dimensional space by place cells and grid cells. Nat. Neurosci. 14, 1182-1188. http://dx.doi.org/10.1038/nn.2892.

Held, R., Dichigans, J., Bauer, J., 1975. Characteristics of moving visual scenes influencing spatial orientation. Vis. Res. 15, 357-365.

Horner, A.J., Bisby, J.A., Zotow, E., Bush, D., Burgess, N., 2016. Grid-like processing of imagined navigation. Curr. Biol. CB 26, 842-847. http://dx.doi.org/10.1016/j.cub.2016.01.042.

Hummel, N., Flanagin, V., 2015. Direction discrimination of self motion consistent optic flow stimuli in multisensory integration cortices. J. Vis. 15, 863. http://dx.doi.org/10. $1167 / 15.12 .863$

Iglói, K., Doeller, C.F., Berthoz, A., Rondi-Reig, L., Burgess, N., 2010. Lateralized human hippocampal activity predicts navigation based on sequence or place memory. Proc. Natl. Acad. Sci. U. S. A. 107, 14466-14471. http://dx.doi.org/10.1073/pnas.1004243107.

Indovina, I., Maffei, V., Lacquaniti, F., 2013a. Anticipating the effects of visual gravity during simulated self-motion: estimates of time-to-passage along vertical and horizontal paths. Exp. Brain Res. 229, 579-586. http://dx.doi.org/10.1007/s00221-013-3620-3.

Indovina, I., Maffei, V., Pauwels, K., Macaluso, E., Orban, G.A., Lacquaniti, F., 2013b. Simulated self-motion in a visual gravity field: sensitivity to vertical and horizontal heading in the human brain. Neurolmage 71, 114-124. http://dx.doi.org/10.1016/j. neuroimage.2013.01.005.

Indovina, I., Mazzarella, E., Maffei, V., Cesqui, B., Passamonti, L., Lacquaniti, F., 2015. Sound-evoked vestibular stimulation affects the anticipation of gravity effects during visual self-motion. Exp. Brain Res. 233, 2365-2371. http://dx.doi.org/10.1007/ s00221-015-4306-9.

Janssen, P., Vogels, R., Orban, G.A., 1999. Macaque inferior temporal neurons are selective for disparity-defined three-dimensional shapes. Proc. Natl. Acad. Sci. U. S. A. 96, 8217-8222.

Janssen, P., Vogels, R., Orban, G.A., 2000a. Three-dimensional shape coding in inferior temporal cortex. Neuron 27, 385-397.

Janssen, P., Vogels, R., Orban, G.A., 2000b. Selectivity for 3D shape that reveals distinct areas within macaque inferior temporal cortex. Science 288, 2054-2056.

Janssen, P., Vogels, R., Liu, Y., Orban, G.A., 2001. Macaque inferior temporal neurons are selective for three-dimensional boundaries and surfaces. J. Neurosci. 21, 9419-9429.

Janssen, P., Vogels, R., Liu, Y., Orban, G.A., 2003. At least at the level of inferior temporal cortex, the stereo correspondence problem is solved. Neuron 37, 693-701.

Jeffery, K.J., Jovalekic, A., Verriotis, M., Hayman, R., 2013. Navigating in a threedimensional world. Behav. Brain Sci. 36, 523-543. http://dx.doi.org/10.1017/ S0140525X12002476.

Jenkinson, M., Bannister, P., Brady, M., Smith, S., 2002. Improved optimization for the robust and accurate linear registration and motion correction of brain images. NeuroImage 17, 825-841.

Kesner, R.P., 2009. The posterior parietal cortex and long-term memory representation of spatial information. Neurobiol. Learn. Mem. 91, 197-206. http://dx.doi.org/10.1016/j. nlm.2008.09.004.

Knight, R., Piette, C.E., Page, H., Walters, D., Marozzi, E., Nardini, M., Stringer, S., Jeffery, K.J., 2014. Weighted cue integration in the rodent head direction system. Philos. Trans. R. Soc. Lond. Ser. B Biol. Sci. 369, 20120512. http://dx.doi.org/10.1098/rstb.2012.0512.

Kravitz, D.J., Saleem, K.S., Baker, C.I., Mishkin, M., 2011. A new neural framework for visuospatial processing. Nat. Rev. Neurosci. 12, 217-230. http://dx.doi.org/10.1038/ nrn3008.

Kriegeskorte, N., Goebel, R., Bandettini, P., 2006. Information-based functional brain mapping. Proc. Natl. Acad. Sci. U. S. A. 103, 3863-3868. http://dx.doi.org/10.1073/pnas. 0600244103.

Lacquaniti, F., Bosco, G., Indovina, I., La Scaleia, B., Maffei, V., Moscatelli, A., Zago, M., 2013. Visual gravitational motion and the vestibular system in humans. Front. Integr. Neurosci. 7, 101. http://dx.doi.org/10.3389/fnint.2013.00101.

Liu, Y., Vogels, R., Orban, G.A., 2004. Convergence of depth from texture and depth from disparity in macaque inferior temporal cortex. J. Neurosci. 24, 3795-3800. http://dx. doi.org/10.1523/JNEUROSCI.0150-04.2004.

Maffei, V., Indovina, I., Macaluso, E., Ivanenko, Y.P., Orban, G.A., Lacquaniti, F., 2015. Visual gravity cues in the interpretation of biological movements: neural correlates in humans. NeuroImage 104, 221-230. http://dx.doi.org/10.1016/j.neuroimage.2014. 10.006 .

Maguire, E.A., Burgess, N., Donnett, J.G., Frackowiak, R.S., Frith, C.D., O'Keefe, J., 1998. Knowing where and getting there: a human navigation network. Science 280, 921-924.
Mast, F., Kosslyn, S.M., Berthoz, A., 1999. Visual mental imagery interferes with allocentric orientation judgements. Neuroreport 10, 3549-3553.

Nitz, D., 2009. Parietal cortex, navigation, and the construction of arbitrary reference frames for spatial information. Neurobiol. Learn. Mem. 91, 179-185. http://dx.doi. org/10.1016/j.nlm.2008.08.007.

Norman, K.A., Polyn, S.M., Detre, G.J., Haxby, J.V., 2006. Beyond mind-reading: multi-voxe pattern analysis of fMRI data. Trends Cogn. Sci. 10, 424-430. http://dx.doi.org/10. 1016/j.tics.2006.07.005.

Oldfield, R.C., 1971. The assessment and analysis of handedness: the Edinburgh inventory. Neuropsychologia 9, 97-113.

Orban, G.A., 2013. Which animal model for understanding human navigation in a threedimensional world? Behav. Brain Sci. 36, 558-559. http://dx.doi.org/10.1017/ S0140525X13000484.

Orban, G.A., Janssen, P., Vogels, R., 2006. Extracting 3D structure from disparity. Trends Neurosci. 29, 466-473. http://dx.doi.org/10.1016/j.tins.2006.06.012.

Peuskens, H., Sunaert, S., Dupont, P., Van Hecke, P., Orban, G.A., 2001. Human brain regions involved in heading estimation. J. Neurosci. 21, 2451-2461.

Peuskens, H., Claeys, K.G., Todd, J.T., Norman, J.F., Van Hecke, P., Orban, G.A., 2004. Attention to 3-D shape, 3-D motion, and texture in 3-D structure from motion displays. J. Cogn. Neurosci. 16, 665-682. http://dx.doi.org/10.1162/089892904323057371.

Phinney, R.E., Siegel, R.M., 2000. Speed selectivity for optic flow in area 7a of the behaving macaque. Cereb. Cortex N. Y. N 1991 (10), 413-421.

Pitzalis, S., Sereno, M.I., Committeri, G., Fattori, P., Galati, G., Patria, F., Galletti, C., 2010 Human v6: the medial motion area. Cereb. Cortex N. Y. N 1991 (20), 411-424. http://dx.doi.org/10.1093/cercor/bhp112.

Riecke, B.E., Sigurdarson, S., Milne, A.P., 2012. Moving through virtual reality without moving? Cogn. Process. 13 (Suppl. 1), S293-S297. http://dx.doi.org/10.1007/ s10339-012-0491-7.

Rubin, A., Yartsev, M.M., Ulanovsky, N., 2014. Encoding of head direction by hippocampal place cells in bats. J. Neurosci. 34, 1067-1080. http://dx.doi.org/10.1523/JNEUROSCI. 5393-12.2014.

Sakata, H., Kusunoki, M., 1992. Organization of space perception: neural representation of three-dimensional space in the posterior parietal cortex. Curr. Opin. Neurobiol. 2, $170-174$.

Save, E., Poucet, B., 2009. Role of the parietal cortex in long-term representation of spatial information in the rat. Neurobiol. Learn. Mem. 91, 172-178. http://dx.doi.org/10. 1016/j.nlm.2008.08.005.

Shelton, A.L., Gabrieli, J.D.E., 2002. Neural correlates of encoding space from route and survey perspectives. J. Neurosci. 22, 2711-2717.

Sherrill, K.R., Erdem, U.M., Ross, R.S., Brown, T.I., Hasselmo, M.E., Stern, C.E., 2013. Hippocampus and retrosplenial cortex combine path integration signals for successful navigation. J. Neurosci. 33, 19304-19313. http://dx.doi.org/10.1523/JNEUROSCI.1825-13. 2013.

Shine, J.P., Valde's-Herrera, J.P., Hegarty, M., Wolbers, T., 2016. The human retrosplenial cortex and thalamus code head direction in a global reference frame. J. Neurosci. 36 (24), 6371-6381.

Stella, F., Treves, A., 2015. The self-organization of grid cells in 3D. eLife 4. http://dx.doi. org/10.7554/eLife.05913.

Sulpizio, V., Committeri, G., Galati, G., 2014. Distributed cognitive maps reflecting real distances between places and views in the human brain. Front. Hum. Neurosci. 8, 716. http://dx.doi.org/10.3389/fnhum.2014.00716.

Taira, M., Tsutsui, K.I., Jiang, M., Yara, K., Sakata, H., 2000. Parietal neurons represent surface orientation from the gradient of binocular disparity. J. Neurophysiol. 83, 3140-3146.

Taube, J.S., Shinder, M., 2013. On the nature of three-dimensional encoding in the cognitive map: commentary on Hayman, Verriotis, Jovalekic, Fenton, and Jeffery. Hippocampus 23, 14-21. http://dx.doi.org/10.1002/hipo.22074.

Taube, J.S., Valerio, S., Yoder, R.M., 2013. Is navigation in virtual reality with FMRI really navigation? J. Cogn. Neurosci. 25, 1008-1019. http://dx.doi.org/10.1162/jocn_a_ 00386.

Tsutsui, K.-I., Sakata, H., Naganuma, T., Taira, M., 2002. Neural correlates for perception of 3D surface orientation from texture gradient. Science 298, 409-412. http://dx.doi. org/10.1126/science.1074128.

Tzourio-Mazoyer, N., Landeau, B., Papathanassiou, D., Crivello, F., Etard, O., Delcroix, N. Mazoyer, B., Joliot, M., 2002. Automated anatomical labeling of activations in SPM using a macroscopic anatomical parcellation of the MNI MRI single-subject brain. Neurolmage 15, 273-289. http://dx.doi.org/10.1006/nimg.2001.0978.

Van Essen, D.C., 2005. A population-average, landmark- and surface-based (PALS) atlas of human cerebral cortex. NeuroImage 28, 635-662. http://dx.doi.org/10.1016/j. neuroimage.2005.06.058.

Vanduffel, W., Fize, D., Peuskens, H., Denys, K., Sunaert, S., Todd, J.T., Orban, G.A., 2002. Extracting 3D from motion: differences in human and monkey intraparietal cortex. Science 298, 413-415. http://dx.doi.org/10.1126/science.1073574.

Vaziri, S., Connor, C.E., 2016. Representation of gravity-aligned scene structure in ventral pathway visual cortex. Curr. Biol. CB 26, 766-774. http://dx.doi.org/10.1016/j.cub. 2016.01.022.

Vaziri, S., Carlson, E.T., Wang, Z., Connor, C.E., 2014. A channel for 3D environmental shape in anterior inferotemporal cortex. Neuron 84, 55-62. http://dx.doi.org/10.1016/j. neuron.2014.08.043.

Vidal, M., Lipshits, M., McIntyre, J., Berthoz, A., 2003. Gravity and spatial orientation in virtual 3D-mazes. J. Vestib. Res. Equilib. Orientat. 13, 273-286.

Vidal, M., Amorim, M.-A., Berthoz, A., 2004. Navigating in a virtual three-dimensional maze: how do egocentric and allocentric reference frames interact? Brain Res. Cogn. Brain Res. 19, 244-258. http://dx.doi.org/10.1016/j.cogbrainres.2003.12.006.

Vidal, M., Amorim, M.-A., McIntyre, J., Berthoz, A., 2006. The perception of visually presented yaw and pitch turns: assessing the contribution of motion, static, and cognitive cues. Percept. Psychophys. 68, 1338-1350. 
Wall, M.B., Smith, A.T., 2008. The representation of egomotion in the human brain. Curr. Biol. CB 18, 191-194. http://dx.doi.org/10.1016/j.cub.2007.12.053.

Webster, M.J., Ungerleider, L.G., Bachevalier, J., 1991. Connections of inferior temporal areas TE and TEO with medial temporal-lobe structures in infant and adult monkeys. J. Neurosci. 11, 1095-1116.

Webster, M.J., Bachevalier, J., Ungerleider, L.G., 1994. Connections of inferior temporal areas TEO and TE with parietal and frontal cortex in macaque monkeys. Cereb. Cortex N. Y. N 1991 (4), 470-483.

Whitlock, J.R., Pfuhl, G., Dagslott, N., Moser, M.-B., Moser, E.I., 2012. Functional split between parietal and entorhinal cortices in the rat. Neuron 73, 789-802. http://dx. doi.org/10.1016/j.neuron.2011.12.028.

Witkin, H.A., Asch, S.E., 1948. Studies in space orientation; further experiments on perception of the upright with displaced visual fields. J. Exp. Psychol. 38, 762-782.

Wolbers, T., Büchel, C., 2005. Dissociable retrosplenial and hippocampal contributions to successful formation of survey representations. J. Neurosci. 25, 3333-3340. http:// dx.doi.org/10.1523/JNEUROSCI.4705-04.2005.

Wolbers, T., Wiener, J.M., Mallot, H.A., Büchel, C., 2007. Differential recruitment of the hippocampus, medial prefrontal cortex, and the human motion complex during path integration in humans. J. Neurosci. 27, 9408-9416. http://dx.doi.org/10.1523/ JNEUROSCI.2146-07.2007.

Woo, C.-W., Krishnan, A., Wager, T.D., 2014. Cluster-extent based thresholding in fMRI analyses: pitfalls and recommendations. NeuroImage 91, 412-419. http://dx.doi. org/10.1016/j.neuroimage.2013.12.058.
Woolrich, M., 2008. Robust group analysis using outlier inference. Neurolmage 41, 286-301. http://dx.doi.org/10.1016/j.neuroimage.2008.02.042.

Woolrich, M.W., Ripley, B.D., Brady, M., Smith, S.M., 2001. Temporal autocorrelation in univariate linear modeling of FMRI data. NeuroImage 14, 1370-1386. http://dx.doi. org/10.1006/nimg.2001.0931.

Woolrich, M.W., Behrens, T.E.J., Beckmann, C.F., Jenkinson, M., Smith, S.M., 2004. Multilevel linear modelling for FMRI group analysis using Bayesian inference. Neurolmage 21, 1732-1747. http://dx.doi.org/10.1016/j.neuroimage.2003.12.023.

Worsley, K.J., 2001. Statistical analysis of activation images (Chapter 14) In: P., J., M., M.P., M., S.S. (Eds.), Functional MRI: An Introduction to Methods. Oxford University Press, New York, NY, pp. 251-270.

Worsley, K.J., Marrett, S., Neelin, P., Vandal, A.C., Friston, K.J., Evans, A.C., 1996. A unified statistical approach for determining significant signals in images of cerebral activation. Hum. Brain Mapp. 4, 58-73. http://dx.doi.org/10.1002/(SICI)1097-0193(1996) 4:1<58::AID-HBM4>3.0.CO;2-O.

Yartsev, M.M., Ulanovsky, N., 2013. Representation of three-dimensional space in the hippocampus of flying bats. Science 340, 367-372. http://dx.doi.org/10.1126/science. 1235338 .

Yoder, R.M., Taube, J.S., 2014. The vestibular contribution to the head direction signal and navigation. Front. Integr. Neurosci. 8, 32. http://dx.doi.org/10.3389/fnint.2014.00032. 